

\section{Las siete maravillas de la Amazonía precolombina, Stéphen Rostain y Carla Jaimes Betancourt (editores), La Paz - Bolivia, Plural editores, 2017}

$\mathrm{C}$ on edición de Stéphen Rostain y Carla Jaimes Betancourt acaba de ser publicado el libro "Las Siete Maravillas de la Amazonía precolombina". El mismo que cuenta con varios artículos de investigadores especializados en el estudio de la selva tropical. Es una publicación de fácil lectura, dirigida al público en general y que tiene como objetivo principal visibilizar la importancia de los sitios arqueológicos, ubicados en esta amplia región que comprende un espacio de más de 7 millones de $\mathrm{km}^{2}$. Lo anterior es un llamado de atención a las instituciones encargadas de velar, proteger y preservar el Patrimonio Cultural de la Humanidad, ya que en sus páginas se pone en evidencia que hasta la actualidad ningún sitio arqueológico de la Amazonía precolombina ha sido incluido en la Lista de Patrimonio Mundial de la UNESCO.

En las reflexiones para explicar el porqué de esta problemática se plantean dos factores fundamentales. En primer lugar, el imaginario a partir del mito de una "selva virgen"; y en segundo término, los modelos teóricos vinculados con el determinismo ambiental que han concebido a las sociedades amazónicas del pasado como grupos humanos limitados por los agentes del bosque húmedo tropical. Esto, a pesar de que hace más de treinta años desde la misma academia ya se han criticado estas conceptualizaciones y se ha realizado un cambio de paradigma en concordancia con la evidencia registrada.

Es importante mencionar que en esta publicación no solo se enlista los sitios arqueológicos encontrados en distintos lugares de la Amazonía, sino que se hace referencia a categorías generales que abarcan una gran cantidad de yacimientos. De este modo, Las Siete Maravillas de la Amazonía se refieren a siete tipos de sitios entre los cuales se encuentran:

- Conchales o concheros.

- Montículos artificiales.

- Campos elevados o camellones.

- Terras pretas.

- Geoglifos.

- Megalitos.

- Arte Rupestre.

El artículo de los concheros o sambaquis a cargo de Francisco Antonio Pugliese Junior, Carlos Augusto Zimpel Neto, y Eduardo Góes Neves, hace referencia a sitios arqueológicos que en su estratigrafía están conformados de manera predominante por conchas. Los mismos que han 
sido identificados en tres zonas principales: 1) El Bajo Amazonas; 2) Estuario y áreas costeras; y 3) Sudoeste amazónico. De acuerdo a los autores en cada una de las regiones los concheros han sido asociados con ambientes en los que existen recursos diversificados y áreas estacionalmente inundables.

En el caso del conchero fluvial Taperinha se han registrado vestigios de cerámica que han sido datadas en más de 7000 años aP. De acuerdo a los autores, estas estructuras empezaron a construirse a inicios del Holoceno, ya que en la base de algunas se han registrado montículos de tierra que corresponden a una primera ocupación. Se ha identificado también que a partir del Holoceno Medio, comienza la acumulación de capas de conchas como material constructivo. En base a los análisis arqueológicos de vestigios botánicos carbonizados, se ha logrado determinar que a partir del Holoceno Medio aumentó de manera significativa el repertorio de plantas manipuladas y la producción sistemática de cerámica. Por lo tanto, en estas estructuras se puede analizar cómo se fueron presentando tres factores que sugieren la intensificación del manejo de estrategias del paisaje: la construcción de estructuras monumentales (se han identificado concheros de más de 6 metros que tienen varios kilómetros de superficie), cultivo de nuevas plantas y producción sistemática de cerámica.

Sobre los montículos artificiales, Heiko Prümers comenta que la gran mayoría de estas estructuras identificadas en la Amazonía son el resultado de ocupaciones durante cientos de años en un mismo lugar. También, menciona de forma muy clara que se han encontrado enterramientos, aunque asociados a contextos domésticos. Se exponen los datos de tres sectores en los que se han identificado montículos artificiales: 1) la isla Marajó en la desembocadura del Amazonas; 2) los Llanos de Mojos en el Norte de Bolivia; y 3) el Valle del Upano en Ecuador.

Los montículos que corresponden a la cultura Marajoara, fechados entre 400 y 1300 d.C., han sido registrados dispersos en los espacios de sabana al noreste de la isla y su ubicación se encuentra en relación con albardones (lomas o fajas de tierra) dejados por paleoríos. Es decir que, la población Marajoara aprovechó estas elevaciones naturales y las modificaron para construir los montículos y mantenerse alejados de las aguas. En cuanto a los montículos de los Llanos de Mojos, éstos se encuentran en las pampas inundables, asociados a terraplenes y canales que comunican una estructura con otra. De igual manera que en la Isla Marajó, algunos montículos de los Llanos de Mojos fueron construidos sobre albardones. Se menciona además, que a través del estudio del material obtenido de excavaciones estratigráficas se ha logrado establecer cinco fases de la Cultura Casarabe, aunque son parte de la misma tradición. Para finalizar, se presentan datos en síntesis (incluidos los antecedentes) de los montículos artificiales del Upano.

Es interesante cómo, tanto en la introducción del libro escrita por Rostain y en este artículo de Prümers se comenta que no han sido publicados hasta la presente fecha los resultados del mapeo mediante LIDAR que fue financiado por el estado ecuatoriano. Pese a este vacío, el autor expone datos de un levantamiento con dron de unos montículos ubicados a pocos kilómetros de la ciudad de Macas, que presentarían una distribución característica compuesta por cuatro plataformas rectangulares que encierran una plaza central dentro de la cual se presenta otro montículo. Finalmente, el autor culmina mencionando que estudios de genética, análisis de isótopos y el estudio de patologías permitirán ampliar el conocimiento de las sociedades que construyeron los montículos artificiales en distintos sectores de la Amazonía.

En el artículo de los campos de cultivo elevados o camellones de autoría de Stéphen Rostain se habla de sitios ubicados en: Bolivia, Venezuela y Guyana. El autor comenta que en algunos casos los campos de cultivo ubicados en las tierras altas sirven para irrigar y concentrar el agua, pero en las tierras bajas, su función, sobre todo, estaba vinculada con el control del exceso de agua a través del drenaje. En el caso de los Llanos de Mojos, se han identificado campos elevados en lugares donde son inexistentes los montículos artificiales. Además, con relación a su forma Rostain menciona que existen: camellones rectangulares y bajos, camellones pequeños, camellones estrechos y campos drenados. En el caso de la Guayana Francesa ha sido identificados en las sabanas inundables, que están ubicadas al oeste de la isla Cayena. Aquí, los análisis de 
laboratorio (muestras de sedimentos y análisis de fitolitos) han arrojado datos que indican que predominaba el cultivo de maíz sobre la yuca en los campos elevados. El autor comenta también, que los cambios ocurridos a partir del primer milenio de la era común sugerirían que tal vez se presentó un aumento demográfico relacionado con el remplazo de la yuca por el cultivo de maíz.

De manera general, uno de los artículos que presenta los datos más novedosos es el que corresponde a las terras pretas. El texto fue escrito por Manuel Arroyo Kalin quien afirma que estas evidencias deben ocupar una posición estelar dentro de las Siete Maravillas de la Amazonía precolombina. Según Arroyo, este tipo de evidencias deben ser consideradas como artefactos arqueológicos debido a su origen antrópico y su relación con antiguos asentamientos indígenas.

Ubicadas en terrenos no inundables cerca de ríos y lagos, o con menor frecuencia en zonas interfluviales vecinas a pequeños cursos de agua, Arroyo define las terras pretas como tierras producto de la acción centenaria de procesos de formación de suelos sobre superficies enriquecidas por las prácticas de habitación de las sociedades precolombinas amazónicas. Entre sus principales características: la oscura coloración de la tierra, el espesor de la capa de suelo orgánico mineral, mayor a la de los otros suelos; tienen altas concentraciones de calcio, fosforo, magnesio, potasio, bario, cobre, manganeso, estroncio, zinc y carbón; además, están asociadas a vestigios arqueológicos cómo: fragmentos de vasijas de cerámica, restos de artefactos de lítica, restos de arcilla calcinada y en algunos casos fragmentos de hueso. Para finalizar, Arroyo Kalin, menciona que las terras pretas son un correlato de un proceso de crecimiento poblacional que pudo haber estado relacionado con una mayor eficiencia en el cultivo de plantas al interior de los asentamientos como en las áreas circundantes. Resulta muy interesante que este tipo de suelos antrópicos tienen un mayor potencial agrícola por su notable fertilidad.

En el artículo escrito Denise Schaan sobre los geoglifos, la autora comenta que estás estructuras son zanjas circundantes ubicadas en enormes recintos geométricos que fueron construidos desde el 1200 a.C. al siglo XVII y que probablemente rodeaban pueblos o campos de cultivo. En Brasil se han registrado estas estructuras en Acre, en los afluentes superiores al río y también en partes altas, lo cual sugiere que posiblemente hayan estado relacionados con actividades defensivas. En el interior de las zanjas se ha registrado poca cerámica y a través de excavaciones se han identificado restos de tablas de madera que tal vez fueron colocados para evitar el desprendimiento de las paredes. En el caso del Acre, Schaan comenta que las zanjas fueron construidas por poblaciones formativas que aún no eran del todo sedentarias. Sin embargo, en Bolivia, en los Llanos de Mojos, las zanjas si se encuentran asociadas a ocupaciones permanentes y también relacionadas con terraplenes y tierras negras.

En cuanto a los sitios megalíticos, los autores Mariana Petry Cabral y Joao Darcy de Moura Saldanha mencionan que en la costa norte de Amapá (Brasil), se han encontrado sitios en los que han sido dispuestas piedras inclinadas de forma asimétrica que dan la impresión de estarse cayendo. Los sitios con estas piedras se encuentran ubicados sobre las cimas de las colinas localizadas cerca de cauces de ríos. En estas elevaciones durante la temporada de lluvia e inundaciones, la cima queda expuesta como una especie de isla. Los autores comentan que en Rego Grande, se encuentra la estructura más grande, la misma que está asociada a pozos para deposición funeraria. En el interior se registraron urnas antropomorfas funerarias. Como un ejemplo ilustrativo hacen alusión a las vasijas completas con pintura polícroma encontradas en el sitio Tucunaré. Este sitio ubicado en la Guyana Oriental ha sido relacionado con la fase cerámica Aristé (2000 a.P.). Al finalizar los autores relacionan las evidencias de megalitos con alineaciones astronómicas lo cual contrasta con la mayoría de artículos del libro en los que se presentan interpretaciones en base a datos duros.

Ya casi finalizando el libro se aborda el tema del arte rupestre considerando que es una de las expresiones simbólicas más antiguas generadas por los seres humanos. Edithe Pereira inicia el artículo haciendo una distinción entre los petroglifos y los pictoglifos. En el caso de los petroglifos, la autora comenta que están localizados preferentemente en las orillas de los ríos y que en algunos casos durante la temporada lluviosa quedan sumergidos. Por otro lado, los 
pictoglifos se encuentran en cuevas alejadas de los ríos y en mayor medida ubicados en las partes altas. Además, menciona que en ningún caso se han registrado evidencias de arte rupestre como parte de un contexto arqueológico cerrado, por lo tanto resulta imposible su datación directa. A pesar de esto en el caso de la Piedra Pintada ubicada en Monte Alegre (Brasil), se ha llegado a realizar una datación indirecta a partir de pigmentos registrados en contexto al interior de la cueva. Las fechas radiocarbónicas arrojaron el dato de 11200 años aP. Dentro de sus principales ilustraciones, se encuentran representaciones humanas completas con rostros expresando gestos. Además de Brasil se hace referencia al arte rupestre de Colombia, Ecuador, Bolivia, Perú, Venezuela, Guyana Francesa, y Suriname. Pereira menciona que en el caso de los petroglifos se pueden identificar motivos recurrentes como son: las figuras antropomorfas completas, las caras las máscaras y las formas geométricas.

Por último, el libro culmina con un artículo teórico a cargo de Philippe Descola. La reflexión del autor cuestiona la existencia de valores y criterios absolutos para definir lo que debe ser considerado como patrimonio o reconocido en el campo de la preservación de bienes. Descola de manera muy clara y a partir del relativismo metodológico de la antropología, pone en evidencia que la lógica a través de la cual se definen los conceptos de los que surge la palabra patrimonio, están vinculados con la modernidad y con el racionalismo ilustrado. Además, en síntesis hace referencia a cuatro tipos de ontologías: naturalista, animista, totemista y el analogismo.

En el caso de la UNESCO, Descola comenta que los criterios a través de los cuales se seleccionan bienes para ser preservados surgen a partir de una epistemología naturalista. Es decir, de un pensamiento en el cual solo los seres humanos conciben que tienen interioridad y que por lo tanto solo ellos son sujetos de derechos. Consecuentemente, en este pensamiento se impone y excluye a las valoraciones que han sido generadas por otras sociedades distintas a la occidental. Cómo un ejemplo, se menciona que para las sociedades amazónicas con una ontología animista, ninguno de los conceptos en los cuales se basa la política de patrimonialización tenían sentido antes de que se vean afectados por el proceso de la explotación de recursos y el etnocidio a partir de las colonias europeas y el neocolonialismo de los estados latinoamericanos. Es decir, que la lógica de la preservación de bienes es parte del mundo moderno, y por lo tanto no existía en el mundo tradicional de las sociedades amazónicas. Descola comenta que a partir de los contactos con agentes externos, los pueblos amazónicos ven que su continuidad cultural se ve amenazada.

En síntesis, el autor expone que la protección y patrimonialización surge a partir de valores particulares que se desarrollan en contextos históricos específicos. Por tal motivo, concluye con una propuesta a la que denomina universalismo relativo en el cual se plantea el respeto al pensamiento del otro y dentro del cual se deben incluir las categorías que son consideradas por los pueblos con epistemologías diferentes a la naturalista.

Para finalizar mencionamos que las "Siete Maravillas de la Amazonía precolombina", es un libro que aporta con información sobre sitios arqueológicos que son parte del patrimonio americano pero que debido a su desconocimiento están en riesgo de ser afectados. Consecuentemente, a partir de lo expuesto en las páginas del libro se busca darlos a conocer para que sepamos de su la importancia. Con lo anterior se realiza un llamado a las instituciones de control para que tomen cartas en el asunto. 